\title{
Evidence-based estimation of insulin resistance
}

\author{
John R. Petrie
}

Received: 23 May 2014 / Accepted: 18 June 2014 / Published online: 17 July 2014

(C) Springer-Verlag Berlin Heidelberg 2014

\begin{abstract}
Insulin resistance is a complex phenotype. Surrogate markers based on peripheral glucose and insulin (and in some cases NEFA) concentrations can provide, at best, moderate approximations to direct physiological measurements (Pearson $r$ values $0.6-0.7$ ). Where the focus is solely on insulin resistance, the evidence reviewed in the paper by Otten et al in this issue (DOI: 10.1007/s00125-014-3285-x) suggests that surrogate markers based on fasting samples alone are as valid as those that require multiple samples and an oral glucose load. This provides an evidence base for simplifying the design of some clinical studies.
\end{abstract}

Keywords Glucose $\cdot$ HOMA $\cdot$ Hyperinsulinaemiceuglycaemic clamp · Insulin · Insulin sensitivity · QUICKI · Surrogate markers
Abbreviations
CVD Cardiovascular disease
OGIS Oral glucose insulin sensitivity
QUICKI Quantitative insulin sensitivity check index

A newcomer to the field of insulin resistance might reasonably wonder: why are there so many ways of measuring it - and which one should I use?

It has been recognised for more than 80 years that resistance to insulin-mediated glucose uptake is key to the pathophysiology of type 2 diabetes - and hypothesised for at least 25 years that impaired insulin action provides an important and early link between metabolic and cardiovascular disease (CVD). However, no consensus has emerged on the relative

\section{J. R. Petrie $(\square)$}

Institute of Cardiovascular and Medical Sciences, BHF Glasgow

Cardiovascular Research Centre, University of Glasgow,

126 University Place, Glasgow G12 8TA, UK

e-mail: john.petrie@glasgow.ac.uk importance of insulin resistance over other pathways thought to be involved in accelerated CVD, or indeed on how these pathways interact. The advent of thiazolidinedione 'insulin sensitisers' provided an intense stimulus to research in the 1990 s, but this was short-lived owing to a protracted period of uncertainty over safety [1].

As interest in insulin-sensitising therapeutic strategies reawakens, the field remains hampered by a bewildering array of surrogate methods of measurement, each with its own formula and acronym. The phrase 'insulin resistance' does not have one simple meaning and covers a range of physiological processes (as discussed recently in a letter published in Diabetologia by Gerald Reaven himself) [2]. It is therefore not surprising that there is no widely accepted and 'user-friendly' standardised method for measuring it, and different laboratories and investigators favour their own methods, rendering comparison of results across the field problematic.

The article by Otten et al in this issue of Diabetologia is therefore both timely and useful [3]. In a careful systematic review the authors have taken a reductionist approach and assessed the performance of the various surrogate measures of insulin resistance against the hyperinsulinaemic-euglycaemic clamp technique as a reference method. The 'clamp' is one of several direct methods for the measurement of insulin resistance, requiring simultaneous intravenous infusion of insulin and glucose over several hours [4]. At steady state, endogenous insulin production is suppressed by a plateau of exogenous hyperinsulinaemia; under these conditions, whole body insulin-mediated glucose uptake approximates the rate of glucose infusion required to maintain plasma glucose at around $5.0 \mathrm{mmol} / 1$ (monitored frequently at the bedside). Depending on the concentration of hyperinsulinaemia achieved, hepatic glucose production can be almost completely suppressed. Therefore, unless a lower dose of insulin is used by design, clamp-derived measurements of insulin sensitivity predominantly reflect peripheral glucose uptake into 
muscle, with higher values indicating greater insulin sensitivity.

There are only a handful of large cohort studies in which insulin resistance has been measured invasively using a clamp or another direct method [5, 6], and these have provided some important insights into key scientific questions. However, in view of the time-consuming and labour-intensive nature of direct methods, surrogate methods are used much more often. All involve measuring insulin and glucose concentrations either in a fasting blood sample or from a series of samples collected during an OGTT. As detailed in the paper by Otten et al [3], such methods have been derived either empirically (from first principles, e.g. HOMA-IR) or by mathematical modelling (statistical analysis of discovery datasets and subsequent validation in independent cohorts, e.g. oral glucose insulin sensitivity [OGIS]).

Otten et al identified 120 published studies over the last 30 years in which surrogate measures of insulin resistance were reported in association with contemporaneous clamp measurements. They extracted data on eight 'fasting' methods and ten OGTT-based methods. In pooling correlation coefficients, they found considerable heterogeneity between studies and evidence of publication bias for some methods. As some studies contained errors, even including ambiguities over the direction of some correlations, they contacted the original authors where possible and also conducted a series of sensitivity analyses.

In all cases, the strength of pooled correlations between surrogate measures of insulin sensitivity and clamp-derived values was at best moderate (Pearson $r$ values between 0.6 and 0.7). This is perhaps not surprising given the complex interaction of processes that determine the insulin and glucose concentrations on which these indices are based (Fig. 1). Confidence intervals for these correlations were overlapping; hence none of the methods examined was definitively better (or worse) than the others.

For insulin resistance to be computed, all surrogate measures rely on detecting high endogenous serum insulin concentrations in the presence of normal or high glucose concentrations. Teleologically, in this situation, beta cells are forced to secrete additional insulin in an effort to drive down glucose levels towards normal (termed 'compensatory hyperinsulinaemia'). In contradistinction to a clamp experiment, in which physiological feedback between glucose and insulin concentrations is disrupted, there is a requirement for preservation of beta cell function. Glucose levels are tightly regulated in healthy individuals, but, nevertheless, equations based on multiplication of fasting insulin and glucose concentrations (e.g. HOMA-IR), or addition of their logarithms (e.g. the quantitative insulin sensitivity check index [QUICKI]), tend to estimate insulin resistance more accurately than fasting insulin concentrations alone. This appears to be the case across subgroups defined by glucose tolerance, even though by definition a similar value can be computed for an insulin-resistant individual with

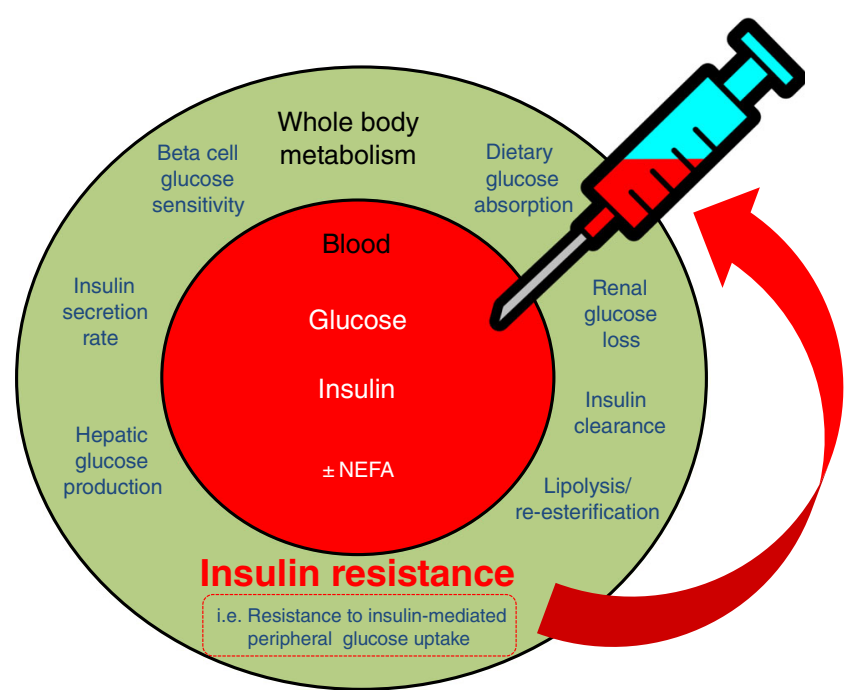

Fig. 1 Whole body glucose metabolism can be divided into a number of components (white text in green circle), all of which can be measured using detailed physiological techniques. Each component exerts an influence on circulating glucose and insulin concentrations at any given moment of blood sampling. Resistance to insulin-mediated peripheral glucose uptake (red box with dotted lines) _ or 'insulin resistance' (red) - is just one component of glucose metabolism; it is measured physiologically during steady-state hyperinsulinaemia using the reference euglycaemic clamp method. All of the surrogate methods for estimating insulin resistance reviewed by Otten et al depend on simple measurements of glucose and insulin (white text in red circle) in fasting or OGTT samples from blood (red circle). As the measurements on which these surrogates are based are influenced by all components of glucose metabolism, and not just the component of interest, they can at best provide an approximation of the true value

hyperinsulinaemia/normal plasma glucose and for an insulin-sensitive individual with impaired insulin secretion/ high plasma glucose.

Otten et al found that the non-OGTT surrogate measure which exhibited the numerically highest pooled correlation coefficient with the reference method was 'revised QUICKI' [3]. This method takes a broader physiological scope than most other methods by incorporating the fasting concentration of NEFA. This approach must add genuine biological value, as higher correlation coefficients with the clamp are observed despite the introduction of an additional source of assay error.

Physiological principles suggest that it should be possible to improve on 'fasting' surrogate measures by exploiting the rich dynamic insulin and glucose data generated across an OGTT. However, Otten et al found no advantage of OGTTbased methods, at least for the purpose of estimating insulin resistance. This is a useful and pragmatic message for researchers focusing on insulin resistance. However, it should be noted that some surrogate methods also generate numerical values for parameters describing other important aspects of carbohydrate metabolism (e.g. insulin secretion rate, beta cell glucose sensitivity). It would be useful if publication of the paper by Otten at al stimulated a further systematic review of indices of beta cell function, whether based on fasting (e.g. 
HOMA-B) or OGTT samples (e.g. OGIS beta cell glucose sensitivity, Stumvoll first-phase index).

Otten et al rightly discuss the quality of the reference clamp data available for their review. There are many aspects of clamp methodology that vary among centres. These include target steady-state insulin level, the type of sample used for glucose assay ('arterialised' or arterial), whether the dose of insulin is calculated according to body weight or surface area, whether the dose of insulin is measured accurately and thoroughly mixed with diluents, the duration of hyperinsulinaemia induced, whether there are one or two 'steps' of hyperinsulinaemia and what degree of error in final glucose concentrations is accepted in applying the 'space correction' (quality control). There are also issues relating to calculation of the result: should glucose metabolised (' $M$ ') be adjusted for the steady-state concentration of insulin ('I') achieved (' $M / \mathrm{I}$ '), and should it be expressed per unit of body mass or per unit of lean body mass (LBM)? There has been some convergence on these issues in recent years in Europe (particularly in the 18 centres taking part in the Relationship between Insulin Sensitivity and Cardiovascular disease [RISC] study) [5, 7, 8], but they have not been resolved on an international basis. It was not therefore a simple matter for the authors to extract the reference clamp data they sought for comparison with the surrogates; again they had to show a number of sensitivity analyses.

As a key conclusion, Otten et al call for large-scale studies aimed at further validating those fasting and OGTT-based indices that they found to have numerically higher correlation coefficients with the reference method [3]. This is a logical suggestion but seems at this time to be no more likely to attract sufficient funding than it has over the previous 30 years. However, one approach attracting current attention is the application of '-omics' technology to screen for novel circulating biomarkers that accurately reflect insulin resistance [9]. Whether such markers could ever replace (or even routinely augment) the best of the current surrogates is an open question, but the area is worthy of exploration -well-validated non-insulin/non-glucose biomarkers of insulin resistance would be invaluable research tools e.g. for studies involving participants already on glucose-lowering therapies. The identity of the relevant proteins or metabolites might also reveal new therapeutic targets. In this context, stored samples from existing cohorts are a useful resource for discovery, but new well-characterised cohorts in which insulin resistance has been measured using both reference and surrogate methods would likely be required for validation.

In the short term, the paper by Otten et al will help researchers to design studies around more reliable surrogate measures and help newcomers in the field to navigate the previous literature (for this purpose, Table 1 is particularly useful). In the longer term, it is hoped that the paper will stimulate the search for better tools for understanding the mechanisms and pathways by which insulin resistance leads to diabetes and the exact nature of its role in the pathogenesis of cardiovascular disease.

Funding The European Group for the study of Insulin Resistance of which the author is current President receives an unrestricted educational grant from Novo Nordisk Europe in support of its annual meeting.

Duality of interest I am an author of references [28] and [66] reviewed in the paper to which this Commentary refers.

Contribution statement The author was the sole contributor to this paper.

\section{References}

1. Hiatt WR, Kaul S, Smith RJ (2013) The cardiovascular safety of diabetes drugs - insights from the rosiglitazone experience. N Engl J Med 369:1285-1287

2. Reaven GM (2013) What do we learn from measurements of HOMA-IR? Diabetologia 56:1867-1868

3. Otten J, Ahren B, Olsson T (2014) Surrogate measures of insulin sensitivity versus the hyperinsulinaemic euglycaemic clamp: a metaanalysis. Diabetologia. doi:10.1007/s00125-014-3285-x

4. DeFronzo RA, Tobin JD, Andres R (1979) Glucose clamp technique: a method for quantifying insulin secretion and resistance. Am J Physiol 237:E214-E223

5. Hills SA, Balkau B, Coppack SW et al (2004) The EGIR-RISC study (The European group for the study of insulin resistance: relationship between insulin sensitivity and cardiovascular disease risk): I. Methodology and objectives. Diabetologia 47:566-570

6. Wagenknecht LE, Mayer EJ, Rewers M et al (1995) The insulin resistance atherosclerosis study (IRAS) objectives, design, and recruitment results. Ann Epidemiol 5:464-472

7. Kozakova M, Natali A, Dekker J et al (2013) Insulin sensitivity and carotid intima-media thickness: relationship between insulin sensitivity and cardiovascular risk study. Arterioscler Thromb Vasc Biol 33:1409-1417

8. Petrie JR, Malik MO, Balkau B et al (2013) Euglycemic clamp insulin sensitivity and longitudinal systolic blood pressure: role of sex. Hypertension 62:404-409

9. Cobb J, Gall W, Adam KP et al (2013) A novel fasting blood test for insulin resistance and prediabetes. J Diabetes Sci Technol 7:100-110 